\title{
Newton-type Schemes via the Method of Generalized Quasilinearization for Volterra Integral Equations of the Second Kind
}

\author{
Cesar Martínez-Garza (Corresponding author) \\ Division of Science \\ The Pennsylvania State University - Berks Campus \\ Tulpehocken Rd., Reading, PA 19610, USA \\ Tel: 1-610-396-6438 E-mail: cxm58@psu.edu
}

\begin{abstract}
In this paper we use the Method of Generalized Quasilinearization to obtain Newton-type comparative schemes to solve the Volterra Integral equation of the Second Kind: $0=f(t, x)+\int_{t_{0}}^{t} K(t, s, x(s)) d s$, which has an isolated zero, $x(t)=r(t)$ in $\Omega$ with $\Omega=\left\{(x, t) \mid \alpha_{0}(t) \leq x \leq \beta_{0}(t), t \in J\right\}, J=\left[t_{0}, t_{0}+T\right], T>0$, where $f(t, x) \in C^{0,2}[J \times \Omega, \mathbb{R}], K(t, s, x) \in$ $C^{0,2}[J \times J \times \Omega, \mathbb{R}]$. Several cases where $f$ and $K$ are convex or concave functions are presented.
\end{abstract}

Keywords: Newton's Method, Generalized Quasilinearization, Integral Equations, Convex Functions

\section{Introduction}

The Method of Generalized Quasilinearization has been recently shown to be successfully employed to solve the equation

$$
0=f(x)
$$

where $f \in C[\mathbb{R}, \mathbb{R}]$, by comparing it to the initial value problem (IVP)

$$
x^{\prime}=f(x), \quad x\left(t_{0}\right)=x_{0},
$$

on $t \in J, J=\left[t_{0}, t_{0}+T\right], T>0$, (Lakshmikantham and Vatsala, 2005; Martìnez-Garza, in press). The Method of Generalized Quasilinearization is a powerful tool to solve nonlinear differential equations (Lakshmikantham and Vatsala, 1998) which combines the concepts of upper and lower solutions with monotone iterative techniques (Ladde, Lakshmikantham, and Vatsala, 1985). By using Generalized Quasilinearization, one can construct a framework comparable to Newton's method to solve for an isolated zero, $x_{0}$ of (1). Newton's method simplicity and rapid convergence renders it as a first choice to solve (1), but its limitations have given rise to a broad body of work that is continuously evolving as seen in Bellman and Kalaba (1965),Ezquerro and Hernández, (1999), Gutiérrez and Hernández (2001), Kelley (1995), Ortega and Rheinboldt (1970), and Potra (1987), to name a few. The generation of Newton-like schemes for the solution to (1) from the method of Generalized Quasilinearization is a logical choice, as Quasilinearization follows a similar methodology to Newton's method although for a somewhat different purpose (Bellman and Kalaba, 1965). The main iteration formula for Newton's Method is

$$
x_{n+1}=x_{n}-\frac{f\left(x_{n}\right)}{f_{x}\left(x_{n}\right)}, \quad n \geq 0,
$$

which requires that $f_{x}(x)$ be continuous and also nonzero in a neighborhood of a simple root of (1). This in turn requires knowledge of the monotone character of $f(x)$. The similarities between Newton's method and standard Quasilinearization are enhanced when expressing (3) in the form

$$
0=f\left(x_{n}\right)+f_{x}\left(x_{n}\right)\left(x_{n+1}-x_{n}\right)
$$

and comparing it with the iterative scheme afforded by Quasilinearization to approximate (2):

$$
x_{n+1}^{\prime}=f\left(x_{n}\right)+f_{x}\left(x_{n}\right)\left(x_{n+1}-x_{n}\right), \quad x_{n+1}\left(t_{0}\right)=x_{0} .
$$

The similarities between the method of Generalized Quasilinearization and Newton's method become self-evident when we look at the Newton-Fourier method as is shown next: let $f$ be defined as in (1), and let $\Omega=\left[\alpha_{0}, \beta_{0}\right]$ be a neighborhood of the simple zero of (1). If $0<f\left(\alpha_{0}\right), 0<f\left(\beta_{0}\right), f_{x}(x)>0$, and $f_{x x}(x)>0$, meaning that $f$ is monotone increasing in $[\Omega, \mathbb{R}]$, then there exist monotone sequences $\left\{\alpha_{n}\right\}$ and $\left\{\beta_{n}\right\}$ that converge quadratically to the simple zero of (1) in $\Omega$. These sequences are generated by the iterative scheme

$$
\begin{aligned}
& 0=f\left(\beta_{n}\right)+f_{x}\left(\beta_{n}\right)\left(\beta_{n+1}-\beta_{n}\right), \\
& 0=f\left(\alpha_{n}\right)+f_{x}\left(\beta_{n}\right)\left(\alpha_{n+1}-\alpha_{n}\right),
\end{aligned}
$$


(Lakshmikantham and Vatsala, 2005). Although two sequences are required, the Newton-Fourier method is not more expensive than the traditional Newton's method since the derivative computations are the same for both iterates (Potra, 1987). Comparatively, consider the IVP (2) with the following conditions: $f_{x}(t, x) \geq 0, f_{x x}(t, x) \geq 0, \alpha_{0}^{\prime} \leq f\left(t, \beta_{0}\right), \beta_{0}^{\prime} \leq f\left(t, \alpha_{0}\right)$, where $\alpha_{0}(t) \leq \beta_{0}(t), t \in J$, and $\alpha_{0}(t), \beta_{0}(t)$ are the upper and lower solutions of (2) (Ladde, Laksmikantham and Vatsala, 1985; Lakshmikantham and Vatsala 1998). The corresponding iterative scheme using Generalized Quasilinearization is:

$$
\begin{array}{ll}
\alpha_{n+1}^{\prime}=f\left(t, \beta_{n}\right)+f_{x}\left(t, \beta_{n}\right)\left(\alpha_{n+1}-\alpha_{n}\right), & \alpha_{n+1}(0)=x_{0}, \\
\beta_{n+1}^{\prime}=f\left(t, \alpha_{n}\right)+f_{x}\left(t, \beta_{n}\right)\left(\beta_{n+1}-\beta_{n}\right), & \beta_{n+1}(0)=x_{0},
\end{array}
$$

where $\alpha_{0} \leq x \leq \beta_{0}$ on $J$. These schemes give rise to sequences of iterates that converge uniformly and quadratically to $x(t)$, the unique solution of (2) on $J$. For complete details refer to Lakshmikantham and Vatsala (1998). If one considers the case where $f$ is monotone decreasing, then the Newton-Fourier method is no longer applicable (Potra, 1987), while the solution of a comparable IVP with $f$ monotone decreasing can be approximated with Generalized Quasilinearization (Lakhsmikantham and Vatsala, 2005).

If $f(t, x)$ can be decomposed as the sum of concave and convex functions, $f(t, x)=F(t, x)+G(t, x)$, where $F(t, x)$ and $G(t, x)$ are convex and concave respectively (in terms of x) for $\Omega=\left\{(x, t) \mid \alpha_{0}(t) \leq x \leq \beta_{0}(t), t \in J\right\}, J=\left[t_{0}, t_{0}+\right.$ $T$ ], $T>0$, then one can exploit the convex properties of $F$ and $G$ with Generalized Quasilinearization to create an iterative scheme that generates monotone sequences that converge quadratically to the solution of (1), see Lakshmikantham and Vatsala, (2005) and Martínez-Garza (in press). The present work applies these notions to the problem (1) where $f$ is a Volterra equation of the second kind, and suitable conditions are placed on $f$ and $K$ according to Davis (1962), and Lakshmikantham and Rao (1995).

\section{Statement of the problem}

Consider the equation

$$
0=f(t, x)+\int_{t_{0}}^{t} K(t, s, x(s)) d s, x\left(t_{0}\right)=x_{0},
$$

where $f \in C^{0,2}[\Omega, \mathbb{R}], K \in C^{0,2}\left[\Omega^{*}, \mathbb{R}\right]$. Here, $\Omega=\left\{(x, t) \mid \alpha_{0}(t) \leq x \leq \beta_{0}(t), t \in J\right\}$, and $\Omega^{*}=\left\{(t, s, x) \mid \alpha_{0}(t) \leq x \leq\right.$ $\left.\beta_{0}(t),(t, s) \in J \times J\right\}, J=\left[t_{0}, t_{0}+T\right], T>0$.

Assume that (10) has an isolated zero $x=r \in\left[\alpha_{0}, \beta_{0}\right]$.

In each case presented below we employ a monotone iterative scheme that generates two monotone sequences $\left\{\alpha_{n}\right\}$ and $\left\{\beta_{n}\right\}$ that will converge from both left and right to $r$, the unique solution of (10). We further show that this convergence is quadratic.

\section{Results I}

Theorem 1. Assume that equation (10), has an isolated zero $x=r \in\left[\alpha_{0}(t), \beta_{0}(t)\right], t \in J$. Further, let both $f$ and $K$ be convex on their respective domains, such that

$$
f_{x x} \geq 0, K_{x x} \geq 0 \text {. }
$$

If the following conditions hold,

$$
\begin{aligned}
& 0<f\left(t, \alpha_{0}\right)+\int_{t_{0}}^{t} K\left(t, s, \alpha_{0}(s)\right) d s, \alpha_{0}\left(t_{0}\right)<x_{0}, \\
& 0>f\left(t, \beta_{0}\right)+\int_{t_{0}}^{t} K\left(t, s, \beta_{0}(s)\right) d s, \beta_{0}\left(t_{0}\right)>x_{0}, \\
& f_{x}(t, x)<0, K_{x}(t, s, x)<0, \\
& \alpha_{0}(t) \leq x \leq \beta_{0}(t),(t, s) \in J \times J,
\end{aligned}
$$

then, there exist monotone sequences $\left\{\alpha_{n}(t)\right\}$ and $\left\{\beta_{n}(t)\right\}$ such that

$$
\alpha_{0}(t)<\alpha_{1}(t)<\cdots<\alpha_{n}(t)<r<\beta_{n}(t)<\cdots<\beta_{1}(t)<\beta_{0}(t), t \in J
$$

which converge quadratically to $r$, the isolated zero of (10). The sequences $\left\{\alpha_{n}(t)\right\}$ and $\left\{\beta_{n}(t)\right\}$ are generated by the 
following iterative scheme:

$$
\begin{aligned}
0= & f\left(t, \alpha_{n}\right)+f_{x}\left(t, \alpha_{n}\right)\left(\alpha_{n+1}-\alpha_{n}\right) \\
& +\int_{t_{0}}^{t}\left[K\left(t, s, \alpha_{n}(s)\right)+K_{x}\left(t, s, \alpha_{n}(s)\right)\left(\alpha_{n+1}(s)-\alpha_{n}(s)\right)\right] d s, \alpha_{n}\left(t_{0}\right)=x_{0}, \\
0= & f\left(t, \beta_{n}\right)+f_{x}\left(t, \alpha_{n}\right)\left(\beta_{n+1}-\beta_{n}\right) \\
& +\int_{t_{0}}^{t}\left[K\left(t, s, \beta_{n}(s)\right)+K_{x}\left(t, s, \alpha_{n}(s)\right)\left(\beta_{n+1}(s)-\beta_{n}(s)\right)\right] d s, \beta_{n}\left(t_{0}\right)=x_{0},
\end{aligned}
$$

Proof. Let $p=\alpha_{0}-\alpha_{1}$, then $p\left(t_{0}\right)<0$. By (12) and (16) with $n=0$,

$$
\begin{aligned}
0<f\left(t, \alpha_{0}\right)+\int_{t_{0}}^{t} K\left(t, s, \alpha_{0}(s)\right) d s-\left[f\left(t, \alpha_{0}\right)+f_{x}\left(t, \alpha_{0}\right)\left(\alpha_{1}-\alpha_{0}\right)\right] \\
-\int_{t_{0}}^{t}\left[K\left(t, s, \alpha_{0}(s)\right)+K_{x}\left(t, s, \alpha_{0}(s)\right)\left(\alpha_{1}(s)-\alpha_{0}(s)\right)\right] d s \\
=-\left[f_{x}\left(t, \alpha_{0}\right)\left(\alpha_{1}-\alpha_{0}\right)+\int_{t_{0}}^{t} K_{x}\left(t, s, \alpha_{0}(s)\right)\left(\alpha_{1}(s)-\alpha_{0}(s)\right) d s\right] \\
0<f_{x}\left(t, \alpha_{0}\right) p+\int_{t_{0}}^{t} K_{x}\left(t, s, \alpha_{0}(s)\right) p(s) d s
\end{aligned}
$$

By (14) we have that $f_{x}(t, x)<0$, and $K_{x}(t, s, x)<0$; we conclude that $p<0$, thus $\alpha_{0}<\alpha_{1}$.

Now, show that $\beta_{0}>\beta_{1}$; let $p=\beta_{1}-\beta_{0}$, so $p\left(t_{0}\right)<0$. From (13) and (17) with $n=0$, we have

$$
\begin{aligned}
& 0< f\left(t, \beta_{0}\right)+f_{x}\left(t, \alpha_{0}\right)\left(\beta_{1}-\beta_{0}\right) \\
&+\int_{t_{0}}^{t}\left[K\left(t, s, \beta_{0}(s)\right)+K_{x}\left(t, s, \alpha_{0}(s)\right)\left(\beta_{1}(s)-\beta_{0}(s)\right)\right] d s \\
&-\left[f\left(t, \beta_{0}\right)+\int_{t_{0}}^{t} K\left(t, s, \beta_{0}(s)\right) d s\right] \\
&= f_{x}\left(t, \alpha_{0}\right)\left(\beta_{1}-\beta_{0}\right)+\int_{t_{0}}^{t} K_{x}\left(t, s, \alpha_{0}(s)\right)\left(\beta_{1}(s)-\beta_{0}(s)\right) d s \\
& 0<f_{x}\left(t, \alpha_{0}\right) p+\int_{t_{0}}^{t} K_{x}\left(t, s, \alpha_{0}(s)\right) p(s) d s
\end{aligned}
$$

Again, by (14) we conclude that $p<0$, making $\beta_{1}<\beta_{0}$.

To show that $\alpha_{1}<\beta_{1}$, we combine (16) and (17), both with $n=0$ :

$$
\begin{aligned}
0= & f\left(t, \beta_{0}\right)+f_{x}\left(t, \alpha_{0}\right)\left(\beta_{1}-\beta_{0}\right) \\
& +\int_{t_{0}}^{t}\left[K\left(t, s, \beta_{0}(s)\right)+K_{x}\left(t, s, \alpha_{0}(s)\right)\left(\beta_{1}(s)-\beta_{0}(s)\right)\right] d s \\
& -\left[f\left(t, \alpha_{0}\right)+f_{x}\left(t, \alpha_{0}\right)\left(\alpha_{1}-\alpha_{0}\right)\right] \\
& -\int_{t_{0}}^{t}\left[K\left(t, s, \alpha_{0}(s)\right)+K_{x}\left(t, s, \alpha_{0}(s)\right)\left(\alpha_{1}(s)-\alpha_{0}(s)\right)\right] d s \\
= & {\left[f\left(t, \beta_{0}\right)-f\left(t, \alpha_{0}\right)\right]+f_{x}\left(t, \alpha_{0}\right)\left[\beta_{1}-\beta_{0}-\alpha_{1}+\alpha_{0}\right] } \\
& +\int_{t_{0}}^{t}\left[K\left(t, s, \beta_{0}(s)\right)-K\left(t, s, \alpha_{0}(s)\right)\right] d s \\
& +\int_{t_{0}}^{t} K_{x}\left(t, s, \alpha_{0}(s)\right)\left[\beta_{1}(s)-\beta_{0}(s)-\alpha_{1}(s)+\alpha_{0}(s)\right] d s
\end{aligned}
$$


The Mean Value Theorem yields the following results:

$$
\begin{aligned}
0= & f_{x}(t, \sigma)\left[\beta_{0}-\alpha_{0}\right]+f_{x}\left(t, \alpha_{0}\right)\left[\beta_{1}-\beta_{0}-\alpha_{1}+\alpha_{0}\right] \\
& +\int_{t_{0}}^{t} K_{x}(t, s, \eta(s))\left[\beta_{0}(s)-\alpha_{0}(s)\right] d s \\
& +\int_{t_{0}}^{t} K_{x}\left(t, s, \alpha_{0}(s)\right)\left[\beta_{1}(s)-\beta_{0}(s)-\alpha_{1}(s)+\alpha_{0}(s)\right] d s,
\end{aligned}
$$

where $\sigma, \eta \in\left(\alpha_{0}, \beta_{0}\right)$. By (11) $f_{x}(t, x)$ and $K_{x}(t, s, x)$ are increasing functions; then, $f_{x}\left(t, \alpha_{0}\right)<F_{x}(t, \sigma)$ and $K_{x}\left(t, s, \alpha_{0}\right)<$ $K_{x}(t, s, \eta)$.

$$
\begin{aligned}
& 0> f_{x}\left(t, \alpha_{0}\right)\left[\beta_{0}-\alpha_{0}\right]+f_{x}\left(t, \alpha_{0}\right)\left[\beta_{1}-\beta_{0}-\alpha_{1}+\alpha_{0}\right] \\
&+\int_{t_{0}}^{t} K_{x}\left(t, s, \alpha_{0}(s)\right)\left[\beta_{0}(s)-\alpha_{0}(s)\right] d s \\
&+\int_{t_{0}}^{t} K_{x}\left(t, s, \alpha_{0}(s)\right)\left[\beta_{1}(s)-\beta_{0}(s)-\alpha_{1}(s)+\alpha_{0}(s)\right] d s \\
&= f_{x}\left(t, \alpha_{0}\right)\left[\beta_{1}-\alpha_{1}\right]+\int_{t_{0}}^{t} K_{x}\left(t, s, \alpha_{0}(s)\right)\left[\beta_{1}(s)-\alpha_{1}(s)\right] d s \\
& 0<f_{x}\left(t, \alpha_{0}\right) p+\int_{t_{0}}^{t} K_{x}\left(t, s, \alpha_{0}(s)\right) p(s) d s
\end{aligned}
$$

Then, $p<0$ and $\alpha_{1}<\beta_{1}$ by (14).

Next, show that $\alpha_{1}<r<\beta_{1}$. Let $p=r-\alpha_{1}, p\left(t_{0}\right)=0$. Since $f(t, r)+\int_{t_{0}}^{t} K(t, s, r(s)) d s=0$ we can combine it with (16) for $n=0$, to obtain the following equation.

$$
\begin{aligned}
0= & f(t, r)+\int_{t_{0}}^{t} K(t, s, r(s)) d s-\left[f\left(t, \alpha_{0}\right)+f_{x}\left(t, \alpha_{0}\right)\left(\alpha_{1}-\alpha_{0}\right)\right] \\
& -\int_{t_{0}}^{t}\left[K\left(t, s, \alpha_{0}(s)\right)+K_{x}\left(t, s, \alpha_{0}(s)\right)\left(\alpha_{1}(s)-\alpha_{0}(s)\right)\right] d s \\
= & {\left[f(t, r)-f\left(t, \alpha_{0}\right)\right]+f_{x}\left(t, \alpha_{0}\right)\left(\alpha_{0}-\alpha_{1}\right) } \\
& +\int_{t_{0}}^{t}\left[K(t, s, r(s))-K\left(t, s, \alpha_{0}(s)\right)\right] d s+\int_{t_{0}}^{t} K_{x}\left(t, s, \alpha_{0}(s)\right)\left(\alpha_{0}(s)-\alpha_{1}(s)\right) d s \\
= & f_{x}(t, v)\left(r-\alpha_{0}\right)+f_{x}\left(t, \alpha_{0}\right)\left(\alpha_{0}-\alpha_{1}\right) \\
& +\int_{t_{0}}^{t}\left[K_{x}(t, s, \mu(s))\left(r(s)-\alpha_{1}(s)\right) d s+K_{x}\left(t, s, \alpha_{0}(s)\right)\left(\alpha_{0}(s)-\alpha_{1}(s)\right)\right] d s
\end{aligned}
$$

Where $v, \mu \in\left(\alpha_{0}, r\right)$. We again appeal to the increasing nature of both $f_{x}$ and $K_{x}$ as follows.

$$
\begin{aligned}
0> & f_{x}\left(t, \alpha_{0}\right)\left(r-\alpha_{0}\right)+f_{x}\left(t, \alpha_{0}\right)\left(\alpha_{0}-\alpha_{1}\right) \\
& +\int_{t_{0}}^{t}\left[K_{x}\left(t, s, \alpha_{0}(s)\right)\left(r(s)-\alpha_{1}(s)\right) d s+K_{x}\left(t, s, \alpha_{0}(s)\right)\left(\alpha_{0}(s)-\alpha_{1}(s)\right)\right] d s \\
= & f_{x}\left(t, \alpha_{0}\right)\left(r-\alpha_{1}\right)+\int_{t_{0}}^{t} K_{x}\left(t, s, \alpha_{0}(s)\right)\left(r(s)-\alpha_{1}(s)\right) d s \\
0< & f_{x}\left(t, \alpha_{0}\right) p+\int_{t_{0}}^{t} K_{x}\left(t, s, \alpha_{0}(s)\right) p(s) d s
\end{aligned}
$$

Once again by virtue of (14) $p<0$, so $\alpha_{1}<r$. In a similar fashion, by combining equation (10) for $x=r$ with (17) for $n=0$ one can show that $r<\beta_{1}$. We can now claim that by induction (15) holds true in light of the fact that we have just shown the validity of the base and the $n=1$ cases. Then, there exist two monotone sequences $\left\{\alpha_{n}(t)\right\}$ and $\left\{\beta_{n}(t)\right\}$ for which $\lim _{n \rightarrow \infty} \alpha_{n}(t)=\tilde{a}=r=\tilde{b}=\lim _{n \rightarrow \infty} \beta_{n}(t)$. It is now clear that $\left\{\alpha_{n}(t)\right\}$ and $\left\{\beta_{n}(t)\right\}$ converge to the isolated zero of (10). Below we demonstrate that this convergence is quadratic.

Let $p_{n+1}=r-\alpha_{n+1}>0$, and $q_{n+1}=\beta_{n+1}-r>0$. First, consider the case $p_{n+1}=r-\alpha_{n}>0$. Combining (10) for $x=r$ and (16) we obtain: 


$$
\begin{aligned}
0= & f(t, r)+\int_{t_{0}}^{t} K(t, s, r(s)) d s-\left[f\left(t, \alpha_{n}\right)+f_{x}\left(t, \alpha_{n}\right)\left(\alpha_{n+1}-\alpha_{n}\right)\right] \\
& -\int_{t_{0}}^{t}\left[K\left(t, s, \alpha_{n}(s)\right)+K_{x}\left(t, s, \alpha_{n}(s)\right)\left(\alpha_{n+1}(s)-\alpha_{n}(s)\right)\right] d s \\
= & {\left[f(t, r)-f\left(t, \alpha_{n}\right)\right]-f_{x}\left(t, \alpha_{n}\right)\left(\alpha_{n+1}-\alpha_{n}\right)+\int_{t_{0}}^{t}\left[K(t, s, r(s))-K\left(t, s, \alpha_{n}(s)\right)\right] d s } \\
& \quad-\int_{t_{0}}^{t} K_{x}\left(t, s, \alpha_{n}(s)\right)\left(\alpha_{n+1}(s)-\alpha_{n}(s)\right) d s \\
0= & f_{x}(t, \gamma)\left(r-\alpha_{n}\right)-f_{x}\left(t, \alpha_{n}\right)\left(\alpha_{n+1}-r+r-\alpha_{n}\right) \\
+ & \int_{t_{0}}^{t}\left[K_{x}(t, s, \lambda(s))\left(r(s)-\alpha_{n}(s)\right)-K_{x}\left(t, s, \alpha_{n}(s)\right)\left(\alpha_{n+1}(s)-r(s)+r(s)-\alpha_{n}(s)\right)\right] d s \\
= & f_{x}(t, \gamma) p_{n}-f_{x}\left(t, \alpha_{n}\right)\left(p_{n}-p_{n+1}\right) \\
& +\int_{t_{0}}^{t}\left[K_{x}(t, s, \lambda(s)) p_{n}(s)-K_{x}\left(t, s, \alpha_{n}(s)\right)\left(p_{n}(s)-p_{n+1}(s)\right)\right] d s
\end{aligned}
$$

The Mean Value Theorem produces the results above with $\gamma, \lambda \in\left(\alpha_{n}, r\right)$. We have also added and subtracted $r$ where necessary to incorporate the expressions $p_{n}=r-\alpha_{n}$ and $p_{n+1}=r-\alpha_{n+1}$. The increasing nature of the derivatives allows us to introduce the following inequalities:

$$
\begin{aligned}
& 0<f(t, r) p_{n}-f_{x}\left(t, \alpha_{n}\right)\left(p_{n}-p_{n+1}\right) \\
& \quad+\int_{t_{0}}^{t}\left[K_{x}(t, s, r(s)) p_{n}(s)-K_{x}\left(t, s, \alpha_{n}(s)\right)\left(p_{n}(s)-p_{n+1}(s)\right)\right] d s \\
& =\left[f_{x}(t, r)-f_{x}\left(t, \alpha_{n}\right)\right] p_{n}+f_{x}\left(t, \alpha_{n}\right) p_{n+1} \\
& \quad+\int_{t_{0}}^{t}\left[K_{x}(t, s, r(s))-K_{x}\left(t, s, \alpha_{n}(s)\right)\right] p_{n}(s) d s+\int_{t_{0}}^{t} K_{x}\left(t, s, \alpha_{n}(s)\right) p_{n+1}(s) d s \\
& \quad 0<f_{x x}(t, \zeta) p_{n}^{2}+f_{x}\left(t, \alpha_{n}\right) p_{n+1} \\
& \quad+\int_{t_{0}}^{t} K_{x x}(t, s, \xi(s)) p_{n}^{2}(s) d s+\int_{t_{0}}^{t} K_{x}\left(t, s, \alpha_{n}(s)\right) p_{n+1}(s) d s,
\end{aligned}
$$

where $\zeta, \xi \in\left(\alpha_{n}, r\right)$ by the MVT. Since $f$ and $K$ are continuous and $\Omega, \Omega^{*}$ are closed with respect to x, we can place the following bounds on their derivatives, taking into account conditions (11) establishing that both $f$ and $K$ are convex, and (14) where $f_{x}$ and $K_{x}$ are both negative.

$$
M_{1}>\left|f_{x x}(t, x)\right|, M_{2}>\left|K_{x x}(t, s, x)\right|, N_{1}>\left|f_{x}(t, x)\right|, \text { and } N_{2}>\left|K_{x}(t, s, x)\right|
$$

In terms of the new bounds, we obtain the following inequality:

$$
\begin{aligned}
0 & <M_{1} p_{n}(t)^{2}+M_{2} \int_{t_{0}}^{t} p_{n}^{2}(s) d s-N_{1} p_{n+1}(t)-N_{2} \int_{t_{0}}^{t} p_{n+1}(s) d s \\
& <M_{1} p_{n}^{2}+M_{2} T \max _{t \in J} p_{n}(t)^{2}-N_{1} p_{n+1}(t)-N_{2} T \max _{t \in J} p_{n+1}(t) \\
& <\left(M_{1}+M_{2} T\right) \max _{t \in J} p_{n}(t)^{2}-\left(N_{1}+N_{2} T\right) \max _{t \in J} p_{n+1}(t) \\
\max _{t \in J} p_{n+1}(t) & <\frac{M_{1}+M_{2} T}{N_{1}+N_{2} T} \max _{t \in J} p_{n}(t)^{2}=A \max _{t \in J} p_{n}(t)^{2}
\end{aligned}
$$

Similarly, for $\beta_{n+1}=\beta_{n}-r>0$ we obtain the estimate

$$
\max _{t \in J} q_{n+1}(t)<\frac{M_{1}+M_{2} T}{N_{1}+N_{2} T} \max _{t \in J} q_{n}(t)^{2}=A \max _{t \in J} q_{n}(t)^{2}
$$

where, $M_{1}, M_{2}, N_{1}, N_{2}$ are the same upper bounds defined by (18). The combination of the results (19) with (20) yields:

$$
\max _{t \in J} p_{n+1}(t)+\max _{t \in J} q_{n+1}(t)<A\left[\max _{t \in J} p_{n}^{2}(t)+\max _{t \in J} q_{n}^{2}(t)\right] .
$$


This inequality establishes the quadratic convergence of the sequences of iterates $\left\{\alpha_{n}(t)\right\}$ and $\left\{\beta_{n}(t)\right\}$. As shown above, $\lim _{n \rightarrow \infty} \alpha_{n}(t)=\tilde{a}=r=\tilde{b}=\lim _{n \rightarrow \infty} \beta_{n}(t)$.

The proof of the theorem is complete.

In the case where $f_{x}(t, x)<0, K_{x}(t, s, x)<0$, Theorem 1 is modified accordingly, as shown below. The proof of the following theorem is omitted as it closely resembles the previous proof.

Theorem 2. Assume that equation (10) has an isolated zero $x=r \in\left[\alpha_{0}, \beta_{0}\right]$ for $f \in C^{0,2}[\Omega, \mathbb{R}], \quad K \in C^{0,2}\left[\Omega^{*}, \mathbb{R}\right]$, as defined above. Let (11) and the subsequent conditions hold:

$$
\begin{aligned}
& f_{x x} \geq 0, K_{x x} \geq 0, \\
& 0>f\left(t, \alpha_{0}\right)+\int_{t_{0}}^{t} K\left(t, s, \alpha_{0}(s)\right) d s, \\
& 0<f\left(t, \beta_{0}\right)+\int_{t_{0}}^{t} K\left(t, s, \beta_{0}(s)\right) d s, \\
& f_{x}(t, x)>0, K_{x}(t, s, x)>0, \\
& \alpha_{0}(t) \leq x \leq \beta_{0}(t),(t, s) \in J \times J,
\end{aligned}
$$

there exist monotone sequences $\left\{\alpha_{n}\right\}$ and $\left\{\beta_{n}\right\}$ such that

$$
\alpha_{0}(t)<\alpha_{1}(t)<\cdots<\alpha_{n}(t)<r(t)<\beta_{n}(t)<\cdots<\beta_{1}(t)<\beta_{0}(t), t \in J
$$

which converge quadratically to $r$, the isolated zero of (10). The sequences $\left\{\alpha_{n}\right\}$ and $\left\{\beta_{n}\right\}$ are generated by the following iterative scheme:

$$
\begin{aligned}
0= & f\left(t, \alpha_{n}\right)+f_{x}\left(t, \beta_{n}\right)\left(\alpha_{n+1}-\alpha_{n}\right) \\
& +\int_{t_{0}}^{t}\left[K\left(t, s, \alpha_{n}(s)\right)+K_{x}\left(t, s, \beta_{n}(s)\right)\left(\alpha_{n+1}(s)-\alpha_{n}(s)\right)\right] d s, \alpha_{n}\left(t_{0}\right)=x_{0}, \\
0= & f\left(t, \beta_{n}\right)+f_{x}\left(t, \beta_{n}\right)\left(\beta_{n+1}-\beta_{n}\right) \\
& +\int_{t_{0}}^{t}\left[K\left(t, s, \beta_{n}(s)\right)+K_{x}\left(t, s, \beta_{n}(s)\right)\left(\beta_{n+1}(s)-\beta_{n}(s)\right)\right] d s, \beta_{n}\left(t_{0}\right)=x_{0},
\end{aligned}
$$

\section{Results II}

The next set of results represents the cases where $f$ is concave and $K$ convex (and viceversa) for (10); the reader is referred to Lakshmikantham and Vatsala (1998) for additional details.

Theorem 3. Let the ongoing assumptions on (10) prevail. Then, given the following conditions:

$$
\begin{aligned}
& f_{x x} \leq 0, K_{x x} \geq 0, \\
& 0<f\left(t, \alpha_{0}\right)+\int_{t_{0}}^{t} K\left(t, s, \alpha_{0}(s)\right) d s, \\
& 0>f\left(t, \beta_{0}\right)+\int_{t_{0}}^{t} K\left(t, s, \beta_{0}(s)\right) d s, \\
& f_{x}\left(t, \alpha_{0}\right)<0, K_{x}\left(t, s, \beta_{0}\right)<0, \\
& \alpha_{0}(t) \leq x \leq \beta_{0}(t),(t, s) \in J \times J,
\end{aligned}
$$

there exist monotone sequences $\left\{\alpha_{n}\right\}$ and $\left\{\beta_{n}\right\}$ such that

$$
\alpha_{0}(t)<\alpha_{1}(t)<\cdots<\alpha_{n}(t)<r(t)<\beta_{n}(t)<\cdots<\beta_{1}(t)<\beta_{0}(t), t \in J,
$$

which converge quadratically to $r$, the isolated zero of (10). The sequences $\left\{\alpha_{n}(t)\right\}$ and $\left\{\beta_{n}(t)\right\}$ are generated by the following iterative scheme:

$$
\begin{aligned}
0= & f\left(t, \alpha_{n}\right)+f_{x}\left(t, \beta_{n}\right)\left(\alpha_{n+1}-\alpha_{n}\right) \\
& +\int_{t_{0}}^{t}\left[K\left(t, s, \alpha_{n}(s)\right)+K_{x}\left(t, s, \alpha_{n}(s)\right)\left(\alpha_{n+1}(s)-\alpha_{n}(s)\right)\right] d s, \alpha_{n}\left(t_{0}\right)=x_{0}, \\
0= & f\left(t, \beta_{n}\right)+f_{x}\left(t, \beta_{n}\right)\left(\beta_{n+1}-\beta_{n}\right) \\
& +\int_{t_{0}}^{t}\left[K\left(t, s, \beta_{n}(s)\right)+K_{x}\left(t, s, \alpha_{n}(s)\right)\left(\beta_{n+1}(s)-\beta_{n}(s)\right)\right] d s, \beta_{n}\left(t_{0}\right)=x_{0},
\end{aligned}
$$


Proof. Let $p=\alpha_{0}-\alpha_{1}$. Combining (28) with (32) with $n=0$ yields:

$$
0<f_{x}\left(t, \beta_{0}\right) p+\int_{t_{0}}^{t} K_{x}\left(t, s, \alpha_{0}(s)\right) p(s) d s
$$

By (27) we understand that $f_{x}$ is decreasing and $K_{x}$ is increasing. With this knowledge and condition (30) we produce the following inequality:

$$
f_{x}\left(t, \beta_{0}\right) p+\int_{t_{0}}^{t} K_{x}\left(t, s, \alpha_{0}(s)\right) p(s) d s<f_{x}\left(t, \alpha_{0}\right) p+\int_{t_{0}}^{t} K_{x}\left(t, s, \beta_{0}(s)\right) p(s) d s .
$$

Which implies that $p<0$ so, $\alpha_{0}<\alpha_{1}$. Likewise, for $p=\beta_{1}-\beta_{0}$ with the aid of (43) with $n=0$ and (29) one can show that $p<0$, thus $\beta_{1}<\beta_{0}$. Subsequently, with $p=\alpha_{1}-\beta_{1}$ subtraction of (25) with $n=0$ from (26) with $n=0$ demonstrates that $p<0$ as shown below.

$$
\begin{aligned}
0 & =f\left(t, \beta_{0}\right)+f_{x}\left(t, \beta_{0}\right)\left(\beta_{1}-\beta_{0}\right)+\int_{t_{0}}^{t}\left[K\left(t, s, \beta_{0}(s)\right)+K_{x}\left(t, s, \alpha_{0}(s)\right)\left(\beta_{1}(s)-\beta_{0}(s)\right)\right] d s \\
& -f\left(t, \alpha_{0}\right)-f_{x}\left(t, \beta_{0}\right)\left(\alpha_{1}-\alpha_{0}\right)-\int_{t_{0}}^{t}\left[K\left(t, s, \alpha_{0}(s)\right)+K_{x}\left(t, s, \alpha_{0}(s)\right)\left(\alpha_{1}(s)-\alpha_{0}(s)\right)\right] d s \\
0 & >f_{x}\left(t, \beta_{0}\right)\left(\beta_{1}-\alpha_{1}\right)+\int_{t_{0}}^{t} K_{x}\left(t, s, \alpha_{0}(s)\right)\left(\beta_{1}(s)-\alpha_{1}(s)\right) d s \\
0 & <f_{x}\left(t, \beta_{0}\right) p+\int_{t_{0}}^{t} K_{x}\left(t, s, \alpha_{0}(s)\right) p(s) d s
\end{aligned}
$$

where we use the MVT and (27). Then, by (34), we obtain the desired conclusion. The same line of reasoning leads us to conclude that $\alpha_{0}(t)<\alpha_{1}(t)<r<\beta_{1}(t)<\beta_{0}(t)$; hence by induction, $\alpha_{0}(t)<\alpha_{1}(t)<\cdots<\alpha_{n}(t)<r<\beta_{n}(t)<\cdots<$ $\beta_{1}(t)<\beta_{0}(t), t \in J$.

Quadratic convergence follows in a similar fashion. Letting $p_{n+1}=r-\alpha_{n+1}>0$, and subtracting (32) from (10) with $x=r$ yields,

$$
\begin{aligned}
0= & f(t, r)+\int_{t_{0}}^{t} K(t, s, r(s)) d s-f\left(t, \alpha_{n}\right)-f_{x}\left(t, \beta_{n}\right)\left(\alpha_{n+1}-\alpha_{n}\right) \\
& -\int_{t_{0}}^{t}\left[K\left(t, s, \alpha_{n}(s)\right)+K_{x}\left(t, s, \alpha_{n}(s)\right)\left(\alpha_{n+1}(s)-\alpha_{n}(s)\right)\right] d s \\
= & f_{x}(t, \sigma) p_{n}-f_{x}\left(t, \beta_{n}\right)\left(p_{n}-p_{n+1}\right) \\
& +\int_{t_{0}}^{t}\left[K_{x}(t, s, \eta(s)) p_{n}(s)-K_{x}\left(t, s, \alpha_{n}(s)\right)\left(p_{n}(s)-p_{n+1}(s)\right)\right] d s \\
< & {\left[f_{x}\left(t, \alpha_{n}\right)-f_{x}\left(t, \beta_{n}\right)\right] p_{n}+f_{x}\left(t, \beta_{n}\right) p_{n+1} } \\
& +\int_{t_{0}}^{t}\left[K_{x}(t, s, r(s))-K_{x}\left(t, s, \alpha_{n}(s)\right)\right] p_{n}(s) d s+\int_{t_{0}}^{t} K_{x}\left(t, s, \alpha_{n}(s)\right) p_{n+1}(s) d s \\
< & -f_{x x}(t, v) p_{n}\left(p_{n}+q_{n}\right)+f_{x}\left(t, \beta_{n}\right) p_{n+1} \\
& +\int_{t_{0}}^{t} K_{x x}(t, s, \mu(s)) p_{n}^{2}(s) d s+\int_{t_{0}}^{t} K_{x}\left(t, s, \alpha_{n}(s)\right) p_{n+1}(s) d s .
\end{aligned}
$$

In the preceding process we have used the mean value property twice with $\sigma, \eta \in\left(\alpha_{n}, r\right)$ and $\nu \in\left(\alpha_{n}, \beta_{n}\right), \mu \in\left(\alpha_{n}, r\right)$. From (34) and (27) we have $f_{x}\left(t, \beta_{n}\right)<f_{x}\left(t, \alpha_{0}\right), K_{x}\left(t, s, \alpha_{n}(s)\right)<K_{x}\left(t, s, \beta_{0}(s)\right)$; also $p_{n}^{2}+p_{n} q_{n}<\frac{3}{2} p_{n}^{2}+\frac{1}{2} q_{n}^{2}$. Then,

$$
\begin{aligned}
0 & <M_{1}\left(\frac{3}{2} p_{n}^{2}+\frac{1}{2} q_{n}^{2}\right)-N_{1} p_{n+1}+M_{2} T \max _{t \in J} p_{n}^{2}(t)-N_{2} T \max _{t \in J} p_{n+1}(t) \\
\left(N_{1}+N_{2}\right) \max _{t \in J} p_{n+1}(t) & <\left(\frac{3}{2} M_{1}+M_{2} T\right) p_{n}^{2}(t)+\frac{M_{1}}{2} \max _{t \in J} q_{n}^{2}(t) \\
\max _{t \in J} p_{n+1}(t) & <\left(\frac{3 M_{1}+2 M_{2} T}{2\left(N_{1}+N_{2} T\right)}\right) \max _{t \in J} p_{n}^{2}(t)+\left(\frac{M_{1}}{2\left(N_{1}+N_{2} T\right)}\right) \max _{t \in J} q_{n}^{2}(t) \\
& =B_{1} \max _{t \in J} p_{n}^{2}(t)+B_{2} \max _{t \in J} q_{n}^{2}(t) .
\end{aligned}
$$


Where the bounds are again defined by (18). Upon letting $q_{n+1}=\beta_{n+1}-r>0$ and following a similar procedure, one completes the quadratic convergence results.

$$
\begin{aligned}
\max _{t \in J} q_{n+1}(t) & <\left(\frac{2 M_{1}+3 M_{2} T}{2\left(N_{1}+N_{2} T\right)}\right) \max _{t \in J} q_{n}^{2}(t)+\left(\frac{M_{2} T}{2\left(N_{1}+N_{2} T\right)}\right) \max _{t \in J} p_{n}^{2}(t) \\
& =B_{3} \max _{t \in J} q_{n}^{2}(t)+B_{4} \max _{t \in J} p_{n}^{2}(t) .
\end{aligned}
$$

The same definitions for the bounds are applied again above. Ultimately,

$$
\begin{aligned}
\max _{t \in J} p_{n+1}(t)+\max _{t \in J} q_{n+1}(t) & <\left(B_{1}+B_{4}\right) \max _{t \in J} p_{n}^{2}(t)+\left(B_{2}+B_{3}\right) \max _{t \in J} q_{n}^{2}(t) \\
& =B\left[\max _{t \in J} p_{n}^{2}(t)+\max _{t \in J} q_{n}^{2}(t)\right],
\end{aligned}
$$

where, $B=\frac{3\left(M_{1}+M_{2} T\right)}{2\left(N_{1}+N_{2} T\right)}$; the proof is complete.

Variations in the increasing/decreasing character of $f(t, x)+\int_{t_{0}}^{t} K(t, s, x(s)) d s$ and in the convexity properties of $f(t, x)$ and $K(t, s, x)$ produce subtle differences that are presented below.

Corollary 1. Assume all the conditions of Theorem 3, but replace conditions (27), and (30) by the new conditions

$$
\begin{aligned}
& f_{x x} \geq 0 K_{x x} \leq 0, \\
& f_{x}\left(t, \beta_{0}\right)<0, K_{x}\left(t, s, \alpha_{0}\right)<0, \\
& \alpha_{0}(t) \leq x \leq \beta_{0}(t),(t, s) \in J \times J .
\end{aligned}
$$

Then, the iterative scheme

$$
\begin{aligned}
0= & f\left(t, \alpha_{n}\right)+f_{x}\left(t, \alpha_{n}\right)\left(\alpha_{n+1}-\alpha_{n}\right) \\
& +\int_{t_{0}}^{t}\left[K\left(t, s, \alpha_{n}(s)\right)+K_{x}\left(t, s, \beta_{n}(s)\right)\left(\alpha_{n+1}(s)-\alpha_{n}(s)\right)\right] d s, \alpha_{n}\left(t_{0}\right)=x_{0}, \\
0= & f\left(t, \beta_{n}\right)+f_{x}\left(t, \alpha_{n}\right)\left(\beta_{n+1}-\beta_{n}\right) \\
& +\int_{t_{0}}^{t}\left[K\left(t, s, \beta_{n}(s)\right)+K_{x}\left(t, s, \beta_{n}(s)\right)\left(\beta_{n+1}(s)-\beta_{n}(s)\right)\right] d s, \beta_{n}\left(t_{0}\right)=x_{0},
\end{aligned}
$$

generates monotone sequences $\left\{\alpha_{n}(t)\right\}$ and $\left\{\beta_{n}(t)\right\}$ satisfying (31) which converge quadratically to the isolated zero of (10).

Corollary 2. Assume that all the conditions of Theorem 3 hold, except (28), (29), and (30), which are replaced by the new conditions

$$
\begin{aligned}
& 0>f\left(t, \alpha_{0}\right)+\int_{t_{0}}^{t} K\left(t, s, \alpha_{0}(s)\right) d s, \\
& 0<f\left(t, \beta_{0}\right)+\int_{t_{0}}^{t} K\left(t, s, \beta_{0}(s)\right) d s, \\
& f_{x}\left(t, \beta_{0}\right)>0, K_{x}\left(t, s, \alpha_{0}\right)>0, \\
& \alpha_{0}(t) \leq x \leq \beta_{0}(t),(t, s) \in J \times J,
\end{aligned}
$$

Then, the iterative scheme (36) - (37) generates monotone sequences $\left\{\alpha_{n}(t)\right\}$ and $\left\{\beta_{n}(t)\right\}$ satisfying (31) which converge quadratically to the isolated zero of (10).

Corollary 3. Let $f$ and $K$ have the same convexity properties in (35) from Corollary 1 , and assume that the remaining conditions of Corollary 2 hold, except for (40), which is replaced by the condition

$$
\begin{aligned}
& f_{x}\left(t, \alpha_{0}\right)>0, K_{x}\left(t, s, \beta_{0}\right)>0, \\
& \alpha_{0}(t) \leq x \leq \beta_{0}(t),(t, s) \in J \times J .
\end{aligned}
$$

Then, the iterative scheme (32) - (33) generates monotone sequences $\left\{\alpha_{n}(t)\right\}$ and $\left\{\beta_{n}(t)\right\}$ satisfying (31) which converge quadratically to the isolated zero of (10). 


\section{Conclusions}

The methods described above represent the means to find isolated roots of Volterra integral equations of the second kind, provided that $f$ and $K$ possess convex properties as presented. It is noteworthy that the present work partially complements the existing body of work where Newton's Method is used to find the solution of integral equations; see Gutiérrez and Hernández (2001), Gutiérrez, Hernández, and Salanova (2004), Hübner (1986), and Hernández and Salanova (2005).

\section{References}

Bellman, R.E. \& Kalaba, R.E. (1965). Quasilinearization and nonlinear boundary value problems. New York: Elsevier. Davis, H.T. (1962). Introduction to nonlinear differential and integral equations. New York: Dover.

Ezquerro, J.A., Hernández, M.A. (1999). On a convex acceleration of Newton's method. JOTA, 100, 311-326.

Gutiérrez, J.M., Hernández, M.A. (2001). An application of Newton's Method to differential and integral equations. ANZIAM, 42, 372-386.

Gutiérrez, J.M., Hernández, M.A., Salanova, M.A. (2004). On the approximate solution of some Fredholm integral equations by Newton's method. Southwest Journal of Pure and Applied Mathematics, 1, 1-9.

Hübner, O. (1986). The Newton method for solving the Theodorsen integral equation. J. Comput. Appl. Math, 14, 19-30.

Hernández, M.A., Salanova, M.A. (2005). A Newton-like iterative process for the numerical solution of Fredholm nonlinear equations. J. Integral Equations Appl., 1, 1-17.

Kelley, C.T. (1995). Iterative methods for linear and nonlinear equations, Philadelphia: SIAM.

Ladde, G.S., Lakshmikantham, V. \& Vatsala, A.S. (1985). Monotone iterative technique for nonlinear differential equations. Boston: Pitman.

Lakshmikantham, V., Rao, M.R.M. (1995). Theory of integro-differential equations. London: Gordan \& Breach.

Lakshmikantham, V. \& Vatsala, A.S. (1998). Generalized quasilinearization for nonlinear problems. Dordrecht, The Netherlands: Kluwer Academic Publishers.

Lakshmikantham, V. \& Vatsala, A.S. (2005). Generalized quasilinearization versus Newton's method. Applied Mathematics and Computation, 164, 523-530.

Martínez-Garza, C. (in press). Extensions to generalized quasilinearization versus Newton's method for convex-concave functions.

Ortega, J.M. \& Rheinboldt, W.C. (1970). Iterative solution of nonlinear equations in several variables. New York: Academic Press.

Potra, F.A. (1987). On a monotone Newton-like method. Computing, 39, 233-246. 